\title{
Some considerations on the use of preparative liquid chromatography in the pharmaceutical industry
}

\author{
G. Mann \\ Schering AG, SH Betrieb, Müller Strasse, Postfach 650311, 13342 Berlin, Germany
}

The demand for products at higher and higher degrees of purity and the greater difficulty of the purification problems encountered (such as the resolution of optical isomers) is more and more forcing the pharmaceutical industry to use Preparative High Performance Liquid Chromatography (PHPLC). The present contribution discusses briefly the situation of the pharmaceutical industry today and its chalenges. With practical examples, it illustrates how PHPLC can compete efficiently with other purification processes like recrystallisation and presents some applications. Examples are also given to illustrate various techniques to make the chromatographic process more rugged and stable.

\section{The current situation of the pharmaceutical industry in Germany}

In the chemical industry and particularly in the pharmaceutical industry, separation, purification and refining processes have always had a very important position. Synthesis and natural processes at best seldom deliver the pure required product.
The quality requirements and expected efficiency of the pharmaceutical final product have risen drastically in the last few years. This development has been initiated and imposed from various different viewpoints: increased knowledge, market demands etc. Coming from this angle, only a few examples will be discussed. It should be stressed that these examples should not be seen as representative of all purification problems, in any way.

In comparison to the eighties, the latest scientific knowledge about metabolic and toxic interaction, as well as the bio-availability of a pharmaceutical active substance have led to greatly changed assessment behaviour. This changed assessment behaviour was not altogether caused by the enormous increase in the performance capability of the analytical procedure, as is so often believed. A variety of different influences and factors have helped to create the current assessment scenario.

Nowadays when considering the assessment and classification of ecological and economical interaction - chemists are operating at a very different environment than they were in the past decades.

In the past decades we have witnessed a restructuration and partly a new orientation of the world markets which have even intensified after the resolution of the East/West economic blocks which were previously at logger-heads. This generally positive trend has also caused fundamental changes in the West European market situation. 
In the chemical industry, as well as in one of its major sections -the pharmaceutical industry-, a fiercely competitive situation has developed between the European states and the former COMECON states. Additional competitors have entered the scene, such as the People's Republic of China, South Korea, and others. To an increasing degree, these states are taking over as low wage countries which can supply the world market with basic chemicals. As far as the pharmaceutical field is concerned, China can be considered as an example.

The Chinese pharmaceutical industry is pressing in on the bulk goods market very strongly, for example with respect to the most important steroid basic compounds. The situation is such today that marketing experts are of the opinion that in a few years China will become the major bulk steroid manufacturer.

This future market scenario clearly shows that the highly industrialised European countries and especially Germany, in the not too distant future will probably not be the production place for such products. It will become necessary that the German pharmaceutical industry, and more generally speaking the European pharmaceutical industry, starts devoting itself to high-tech products at a considerably higher extent than before.

The most interesting combination from an economic point of view is a high-tech product with a high added value. On the other hand, high-tech products are R\&D intensive, which also means capital intensive. Vice-versa, it means that the high capital expenditure for R\&D must not be completely passed into the production of a high-tech product. The product has to be marketable from the point of view of performance and price formation.

\section{Performance requirements in the pharmaceutical industry}

The required product purity of $>99 \%$ is, as a rule, the standard today. However, it can be observed that the trend is moving towards an even higher purity. In this respect, it is important to keep in mind the following points:

- The structure and toxicological behaviour of the impurities must be known. It means that all impurities should be identified.

- If there are unknown impurities present, they are not allowed to be at a level higher than $0.1 \%$. In the near future, a lowering of this limit value to $0.05 \%$, or even less, is expected. The admissible total amount of impurities, at a value of approximatively $0.5 \%$, is discussed in a "relatively vague" way.

However, there are exceptions to these rules. For example, compounds which are suspected of having mutagenic or carcinogenic effects, have no limit value. This means that they have to lie below the limit of detection.

There are also more "generous" exceptions, however. For example, for compounds which are analogue to the end product, if they are proven toxicologically harmless, higher concentrations are acceptable. Such cases, however, must be regarded as exceptions.
The testing and admission regulations of the national and European admission authorities demand, besides the many other investigations, long term stability tests e.g., with a product that shows a fingerprint as it is described in the submission file. If this fingerprint changes in the future production phase, then follow-up investigations will be necessary. In the worst case, even new toxicological tests and new long term stabilities will be required. For a long term stability test, one should consider 1.5 to 2 years of labour and investigations. This situation forces the pharmaceutical industry to constantly produce the product quality that has previously been defined in the Drug Master Files (DMF). This is often not so easy to achieve and can be illustrated by the following "everyday" situation.

Consider a final compound which is obtained after a rather large number of synthesis steps. It can happen that, for some reasons, the synthesis route deviates from what it should normally be. Every chemist in a pharmaceutical world knows at least 99 reasons why such a deviation from the plan could come up. Assume, for instance, that the cooling system of the reactor does not perform as expected. Despite the most modern technology and control of the whole chemical reaction through a process control system, it still is not successful in arresting the reaction, at this quality determined stage, at the intended temperature level. There is not sufficient cooling energy available and the reactor runs off at $-5{ }^{\circ} \mathrm{C}$ instead of the desired $-15{ }^{\circ} \mathrm{C}$. Consequently, one of the side reactions runs more extensively than intended; and the corresponding by-productwhich is present at $1.5 \%$ to $2 \%$ with a normal reaction runis now present at $10 \%$. The reaction now not only generated the final product but has also created a problem!

In the pharmaceutical industry, recrystallisation is one of the main purification methods. In the present situation, assume that the manufacturing process stipulates a recrystallisation step. This recrystallisation will very likely not be successful because of the clearly too high level of the relevant by-compound: the specifications state a limit value of $0.2 \%$ and the actual value lies at $1.1 \%$.

Any modification of the recrystallisation method -e.g. other solvents- is not possible. For regulatory reasons any kind of deviation from the manufacturing regulations submitted to the authorities is forbidden.

The company chemist who is responsible in such a situation, uses the only legal means possible: he approves the repetition of the already planned, registered and approved recrystallisation; but the recrystallisation has to be done two times more in order to prevent concentration values falling below the specification limits.

The number of recrystallisation steps is then three instead of the predicted one. The yield loss is about 35\%. Let us now assume that the product is worth $20000 \mathrm{DM} / \mathrm{kg}$ and the standard batch size is $200 \mathrm{~kg}$. It is simple to calculate what the losses are big. This situation is not an unusual case. It occurs in all pharmaceutical companies in Europe and the USA that the author has had an opportunity to visit.

At Schering, one has learned from the situation fictively portrayed here (but also based on a real example) and PHPLC (Preparative High Performance Preparative Chromatography) has replaced crystallisation (in some 
particular cases) as a more economical and more efficient technique.

As mentioned before, when considerably more high-tech products than before we dealt with, then completely different situations (as far as purification processes are concerned) have to be faced. At Schering, as in other pharmaceutical companies where the same situation can be observed, there are high tech products on-line and in the R\&D pipeline. Most of these products have high purification requirements because of the more complex and more demanding chemistry involved. These higher purification requirements have frequently arisen because in those cases the classical purification methods not only had a worse success rate but showed often no success whatsoever.

Other issues must also be kept in mind. For instance, the FDA has announced that a racemic substance will no longer be accepted in the admittance test procedure for drugs in the near future. As yet, the FDA has only accepted the clinical and toxicological investigations and the later marketing of racemic compounds. In the debate regarding racemic mixtures versus optically pure compounds, the drug Contergan ${ }^{\circledR}$ often crops up as an example. The first real sleeping drug that led to the terrible, notorious foetal deformities, was a racemate. The Plus-form had safe sleep-inducing effects whereas the Minus-form caused deformities in the foetus. According to today's knowledge in the field, the opinion is that one can test and assess the effects of a racemate very well. However, the FDA regulations, as well as the National and the European authorities (which are becoming more and more comparable to the USA standards), set the law in the pharmaceutical industry. That means, in other words, that the separation of optical isomers will take a substantially more significant role in separation techniques. Chiral phases and SMB (Simulated Moving Bed) are key words in this field.

\section{Case studies of chromatographic separation processes}

\section{Crystallisation versus PHPLC}

It is clear that an efficient, economical as well as simple crystallisation methods would hardly prompt the demand for any change to an alternative separation technology. However, our experience has told us that many crystallisation processes are far more complex than they initially appear. In these very cases, PHPLC can be an alternative. One of our current separation problems can probably illustrate this.

This product has already been in production for many years. The combination of crude materials and the special properties of the by-compounds frequently cause problems, however. In order to guarantee good and stabilised quality of the final product, three purification stages were necessary. First, a basic pre-purification using gravity column was carried out. It was followed by two crystallisation steps. The average result of these operations are shown below.

\begin{tabular}{lcc}
\hline Purity & Recovery & Total Purification Cost \\
\hline $97.5-98.6 \%$ & $75-78 \%$ & $494 \mathrm{DM} / \mathrm{kg}$ \\
\hline
\end{tabular}

The modest recovery rate and the low purity prompted us to investigate deeper the purification process. We developed a step by step purification method using PHPLC technology exclusively. The results are shown in the table below.

\begin{tabular}{lcc}
\hline Purity & Recovery & Total Purification Cost \\
\hline $99.5-99.7 \%$ & $95-96 \%$ & $786 \mathrm{DM} / \mathrm{kg}$ \\
\hline
\end{tabular}

A closer examination of the results suggests the following comments:

- the purity is significantly increased by using PHPLC (limitation of specification $>98 \%$ ),

- the recovery ratio is considerably higher,

- the production costs is higher (about 59\%), taking into consideration the throughput.

However, if one considers the value of the product (about $20000 \mathrm{DM} / \mathrm{kg}$ ) and the recovery ratio, PHPLC becomes to be more economical than crystallisation, as shown below. The savings occur as follows:

- the recovery ratio of the crystallisation process is $78 \%$ at the maximum,

- the recovery ratio of the PHPLC process is $95 \%$ in the worst case.

The difference in recovery ratio is then at least $17 \%$. For one kilogram of product processed, this represents a saving of about 3400 DM.

Although the PHPLC method is 292 DM/kg more expensive than the established crystallisation process, it produces $0.170 \mathrm{~kg}$ more product per kilogram processed. The corresponding cost saving is then $3108 \mathrm{DM} / \mathrm{kg}$.

\section{Increasing separation performance by using recycling technology}

The following example illustrates a demanding separation problem: the separation of 2 stereoisomers. For this separation, a maximum separation performance of the chromatographic bed is needed.

If, as in the separation problem discussed before, the desired degree of purification performance has not been achieved despite the use of high performance material and the very critical assessment of the packing quality; it is then often possible to use the recycling approach. With this approach, it is not the recycling of crude material but rather the re-injection of a peak (or peak cut) which is made. In order to be prompted to do this, however, the design of the start-chromatograms has to be developed accordingly.

This recycling technique is illustrated in the chromatogram in figure 1 .

\section{Increasing the loading capabilities by peak shaving}

In preparative chromatography in general and in production chromatography in particular, economical considerations dictate to depart from the so-called "baseline resolution" so important in analytical HPLC. In order to significantly increase the throughput per cycle, the chromatographic system is heavily overloaded and the peaks overlap more or less. By targeted peak splitting (that means extracting a 


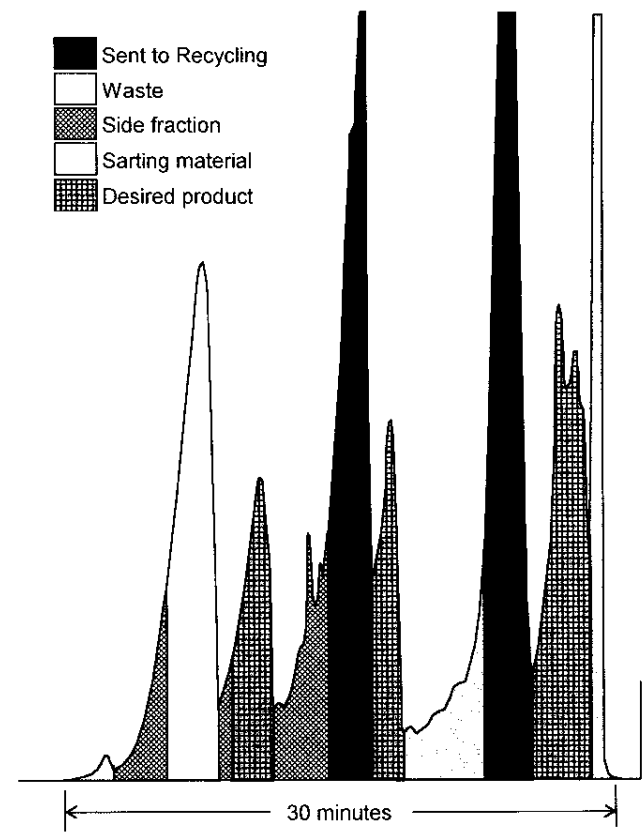

Figure 1. Recycling mode.

multitude of components from the peak profile) and following individual fractions analysis, the exact individual peak profiles can be determined. On the basis of this information the exact fraction cut can be carried out. An example is shown in figure 2 .

\section{Loading and throughput increasing using PAU (Pre Adsorption Unit) and the subsequent interlocking mode}

It is often the case that the crude material to be purified contains an extremely large amount of by-compounds. This, as a rule, and apart from numerous other things, leads to two major problems:

- Upon increasing number of crude injections, so-called "residents" which cannot be removed with the eluent used for the separation accumulate in the chromatographic column. It is often the case that such by-compounds cannot be completely removed by using pre-columns. Consequently, the are chances that the purification column gets slowly fouled, with adverse effects on its separation power. It may become impossible to use the column in a permanent operation.

- If the amount of by-products is too high, then the chromatography column is impeded with an unnecessary load of material, along with concomitant losses of separation performance.

With the help of a kind of pre-adsorption process (PAU, PreAdsorption Unit), it is often relatively simple to remove one or several by-compounds - or at least to reduce their amount quite drastically. Such PAUs should not be considered as a pre-columns.

The production rate rises by the amount of undesired material which is removed by pre-adsorption. When the PAU concept is applied to the previous example the following results are obtained.

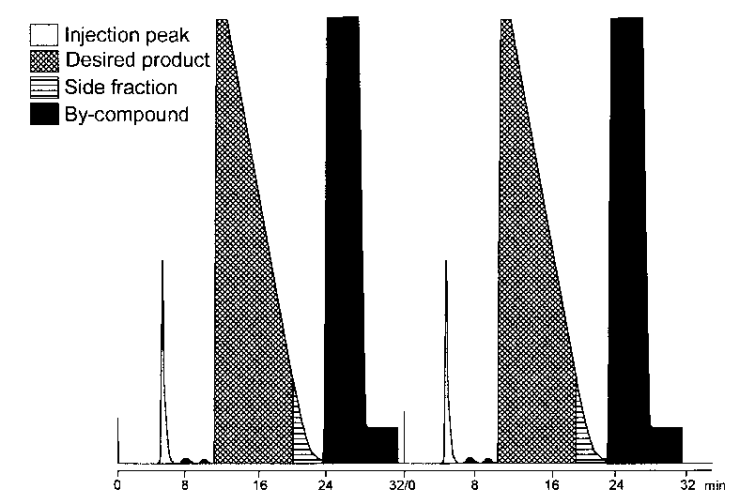

Figure 2. Peak-shaving.

PAU data (related to given example).

\begin{tabular}{lccc}
\hline Unit & Throughput (1) & $\begin{array}{c}\text { Production rate } \\
\text { (without PAU) }\end{array}$ & $\begin{array}{c}\text { Production rate } \\
\text { (with PAU) }\end{array}$ \\
\hline separation & $2800 \mathrm{~kg}$ & $0.770 \mathrm{~kg}$ & $0.845 \mathrm{~kg}$ \\
day & $115200 \mathrm{~kg}$ & $31600 \mathrm{~kg}$ & $34800 \mathrm{~kg}$ \\
week & $576000 \mathrm{~kg}$ & $158000 \mathrm{~kg}$ & $173800 \mathrm{~kg}$ \\
month & $2304000 \mathrm{~kg}$ & $632.20 \mathrm{~kg}$ & $695400 \mathrm{~kg}$
\end{tabular}

(1) The throughput is not related to the crude material composition. (2) On the basis of a separation made on a LC.300 Prochrom column (300 mm internal diameter) and extrapolated to an LC.800 column.

(3) On the basis of a separation made on a LC.50 Prochrom column (50 $\mathrm{mm}$ internal diameter) and extrapolated to an LC.800 column.

It is important to point out that the figures given in the previous table are not the result of calculations, but rather the numbers obtained in practice (with a $300 \mathrm{~mm}$ and a $50 \mathrm{~mm}$ internal diameter columns) and extrapolated to a PHPLC system equipped with a column of $800 \mathrm{~mm}$ internal diameter.

The effect of PAU treatment is shown in figures 3 and 4 for two consecutive injections of crude material. Figure 3 shows the chromatogram of the crude before PAU treatment, and figure 4 after PAU treatment. Through this technique, more "empty" space is produced between the two chromatograms. This space can be used efficiently for overlapping injections: the next injection is made before the previous one is completely eluted (overlapping mode, see Fig. 5). This procedure reduces the effective duration of a run, with concomitant increase in the production rate and decrease in solvent consumption and purification cost.

\section{Shortening the cycle duration and increasing the life of a PHPLC packing material by implementation of a back-flush step}

As has already been mentioned in some of the previous examples, the crude material to be purified by PHPLC often contains undesirable products which, under the chromatographic conditions chosen for the purification, elute very 


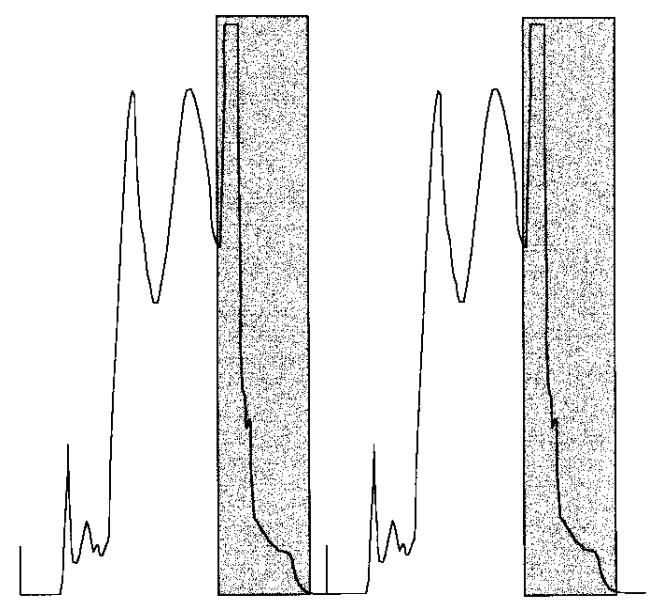

Figure 3. Chromatogram of crude before PAU treatment.

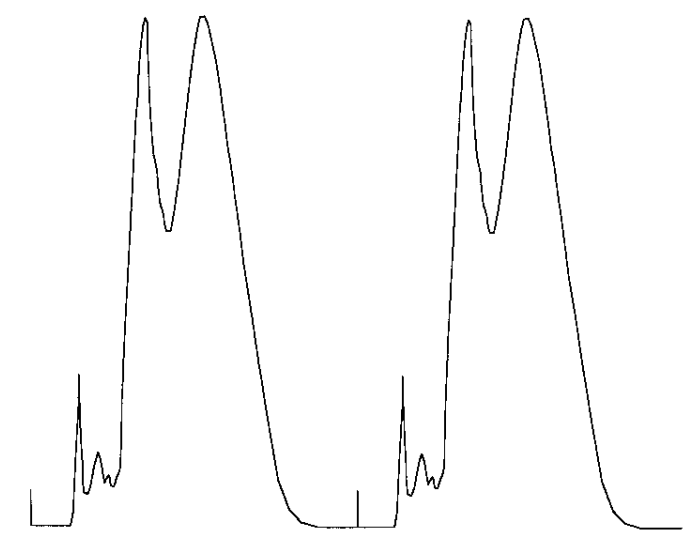

Figure 4. Chromatogram of crude after PAU treatment.

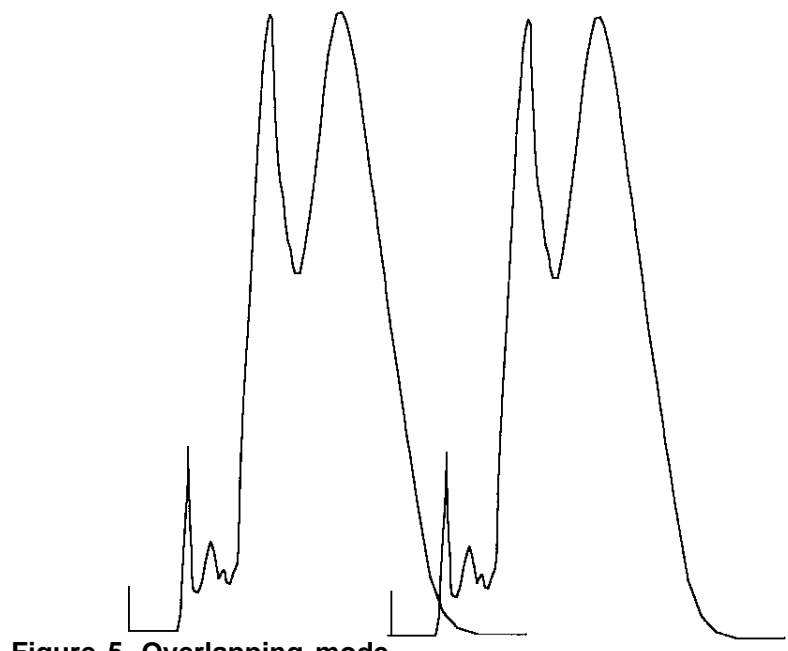

Figure 5. Overlapping mode.

slowly or not at all. This undesirable material can be by-compounds, excesses of reagents or the respective products can be the remaining auxiliary materials. These materials can originate from the reaction itself or from the subsequent processing.
In the process chromatography requirements list, alongside the main target of purity and shortest possible labour time, also stands the longest possible life-time of the chromatographic bed. This is a very important point, and not only for economical reasons. That is why "residents" should be eliminated. The possibilities of pre-purification of the crude material previously mentioned (pre-columns and PAU) are not completely effective in every case. This is regrettable because one can observe situations in which "residents", even present in very small amounts, will cause a collapse in a chromatographic bed.

The most extreme case we encountered was with a column of $300 \mathrm{~mm}$ internal diameter and $25 \mathrm{~cm}$ length packed with $11.6 \mathrm{~kg}$ of silica. The presence of $1.1 \mathrm{~g}$ only of "residents" accumulated over 50 cycles caused an extreme pressure increase and a drastic decrease of selectivity. The $1.1 \mathrm{~g}$ amount of "residents" only penetrated the bed about $0.5 \mathrm{~cm}$, corresponding to a contamination of the bed of about $2 \%$ of its length (basically, the "residents" were trapped at the column inlet). This small contamination was sufficient, however, to render the column almost useless. Frequently, the implementation of a back-flush step can eliminate this very unpleasant situation.

\section{Performance requirements}

The synthesis, often via a large number of steps, does not always lead to a pure end-product. This predicament calls for the application of more or less (and more than less, as a general rule!) costly purification operations. Costly is equal to cost intensive.

The purification step is quite often the most costly step in the production of a pharmaceutical product. Accordingly, it is desirable to reduce as much as possible the purification costs. This should be the short and medium term targets for the future.

The performance requirements in separation technology in the area of product refining unfortunately cannot be defined with only a few comprehensible figures, values and curves.

However, the afore-mentioned processes, needs and scenes create just from themselves, a requirement profile manifested in the points emphasised below:

- the highest possible degree of purity (namely the highest purity the product of interest demands).

- the highest possible stability of the separation process. This means absolute stability and reproducibility over several purification cycles. From today's point of view, this does not mean 40 or 50 cycles, but rather 1000 cycles.

- the highest possible economy of separation processes which has a measured balance of the basic points mentioned before.

- the solvents and auxiliary materials used in the separation process must comply with today's environmental policies. When this is not the case, for instance if methylene-chloride has to be used, then additional equipment is required. In order to attain the current environmental demands, it 
is necessary to acquire special performances and technical installations. This all adds up to a considerable cost burden.

Perhaps initially these 4 points, which only serve to describe the main problem area - special applications demand additional focus points - seem to look like the quadrature of a circle. Established separation processes, show that these problem areas can be resolved.

\section{Separation technology, theory and modelling}

For chromatographic separations of substances, the age of chromatography was initiated by Michail Tswett in 1905 (Fig. 6), apart from a few side issues occurring at certain points in the $18^{\text {th }}$ and $19^{\text {th }}$ centuries. 1905 can be seen as the starting point of chromatography.

Until the late $60 \mathrm{~s}$, these developments were conducted nearly exclusively from empirical data (in very few cases scientifically theoretical) which even by today's standards was really quite revolutionary.

In the ensuing period of radical change, theories and models were initiated and the foundations for the current period were established.

This development of the last 2 decades is due to the work of individual people and groups at colleges and universities, and in the industry as well. Some really pioneering work was achieved by this circle within this period of time. However as mentioned before, it was exclusively due to scientific commitment as well as scientific and economic influenced commitment, of those in industry. This led to formation of isolated solutions.

As a rule, universities and colleges lack the financial basis to enable them to work on complex and cost intensive projects.

In wide areas of industry, product purification and also chromatographical processes are considered part of the whole procedure. Thus, they are incorporated into the company's trade secrets. Any valuable know-how is therefore obviously not accessible to the public.

The first cautious trend towards change, started at PREP'90 where a few colleagues from the industrial sector introduced their work for the first time. In the course of the last 5 years the trend has strengthened so that today a more balanced relation of representatives from industry and universities can be seen at international conferences.

Nevertheless this does not reflect the degree of co-operation. Even today common projects, research etc., bear the traits of only individual efforts.

In order that we can resolve these very complex, fundamental tasks that lie before us, the co-operation between industry and institutions of higher education must be placed on a wider basis.

\section{Stationary phases}

The following points should be kept in mind:

- Economic PHPLC separations need high selectivities, high loading factors and long life-time of the chromatographic bed. The chromatographer increasingly needs high performance packing materials, particularly when

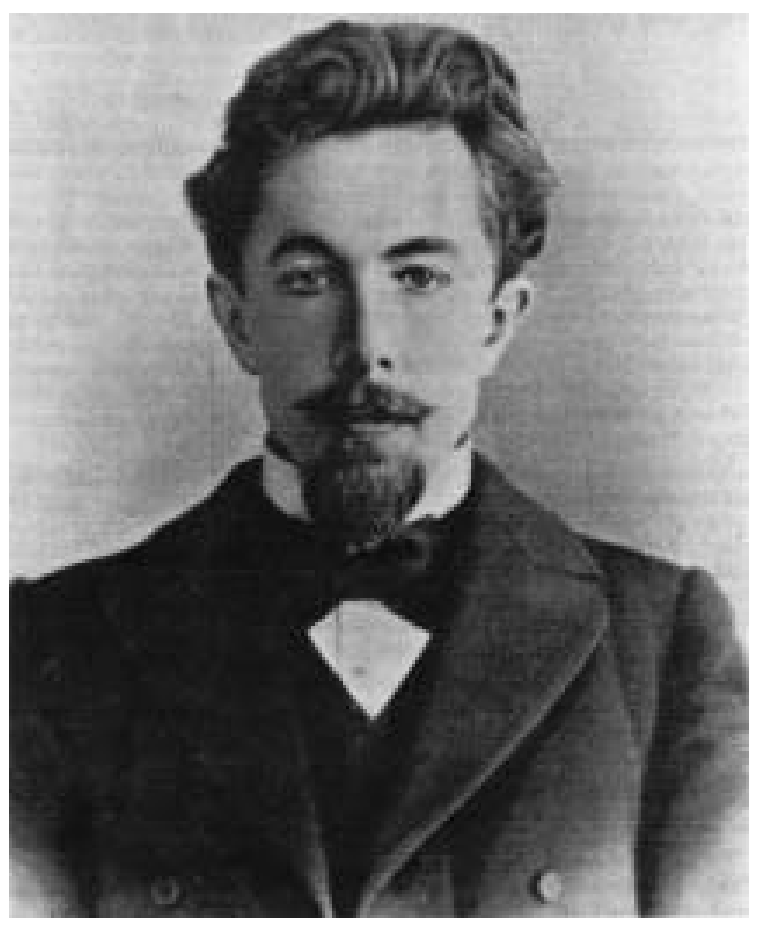

Figure 6. Michail Tswett.

the pressure exerted by process cost increases more and more. The trend is already underway, and in the last 4-5 years, it has been seen that the demand for media corresponding to this criteria has enormously increased. Some manufacturers of separation media have recognised this demand and reacted accordingly. However, from the point of view of the user, one can regret that, at the moment, the number of such manufacturers is still very small.

- For specific processes and for certain throughput amounts, tailor-made media will make sense in the future.

- Chiral separations are becoming more and more important at the production scale, for reasons discussed before. Separation media must be manufactured and marketed considerably cheaper in the future than they are today. Also, these materials must become significantly more stable. As an example, one can just mention the "bleeding" effect of many chiral phases.

- The further development of media based on polymers (and possibly also zeolythes) has not sufficiently been recognised as of today.

- This list only addresses some issues and, in no way, should be seen as complete.

\section{Equipment technology/Sytems engineering}

As already discussed, the purity requirements related to pharmaceutical drugs result in the fact that PHPLC is employed in many important product classes as purification technology. Thus, for e.g. steroids, diagnostics, ß-carbolines, prostanes are purified by chromatography at industrial scale, as well as proteins and peptides. 
As a basic prerequisite, the process chromatographer needs:

- high separation performance,

- adjustable bed lengths (to optimise column performance).

The chromatographic beds should be stable and show reproducible and high performance over long periods of time. The chromatographic equipment must be able to be in continuous operation for several months.

CIP (Cleaning In Place), qualified equipment according to ISO.9000 and automatically generated documentation nowadays should be part of the standard basic equipment. Equipment systems are needed which allow upscaling of up to several tonnes per annum.

The afore-mentioned points do not represent a requirement specification but only address some of the most important issues.

Considering these criteria, currently only two (!!) companies out of a large number of chromatographic equipment manufacturers in the world qualify as suppliers of PHPLC equipment that:

- are suitable for a permanent operation maintaining high separation performance and,

- comply with FDA requirements.

However, these two companies build their equipment with commercially available components as much as possible.
That means functional parts which are not exclusively designed for chromatographical use. Only completely PHPLC specific equipment parts which are not available on the market are exclusively designed and manufactured.

However we could imagine, and it also makes sense that in the near future this same step could follow in PHPLC, what in the analytical HPLC began at the end of the sixties. For example to design components such as pumps, valves etc., which specifically relate to the needs of PHPLC.

The very complex topic of innovative separation technology is naturally not only covered and displayed by chromatographic processes. Techniques other than chromatographic ones should be considered when addressing a purification problem. This was considered when evaluating the present situation and the subsequent performance requirements for the pharmaceutical industry.

Since PHPLC is currently among the most advanced, and at least in particular areas, an already established separation technology that can be found at Schering; the author has restricted himself to describing only case studies coming from this area.

PHPLC related techniques such as SMB and SFC (Supercritical Fluid Chromatography) in specific cases offer some interesting alternatives. Membrane technology, process gas chromatography, crystallisation processes in supercritical areas, just to mention a few other possibilities, show innovative potential as well. 